\section{Combined multiphoton microscopy and optical coherence tomography using a 12-fs broadband source}

\author{
Shuo Tang \\ Tatiana B. Krasieva \\ University of California \\ Beckman Laser Institute \\ Laser Microbeam and Medical Program \\ Irvine, California 92617 \\ Zhongping Chen \\ Bruce J. Tromberg* \\ University of California \\ Beckman Laser Institute \\ Laser Microbeam and Medical Program \\ Irvine, California 92617 \\ and \\ University of California \\ Department of Biomedical Engineering \\ Irvine, California 92617
}

\begin{abstract}
A 12-fs broadband (100-nm) source is used to combine multiphoton microscopy (MPM) and optical coherence tomography (OCT) in a single platform. An ultrafast Ti:sapphire laser simultaneously provides short pulses necessary for efficient MPM excitation and the broad bandwidth required for high-resolution OCT. Using $0.3-\mu \mathrm{m}$ microspheres and a $63 \times, 0.95$ numerical aperture objective, we demonstrate that MPM and OCT channels are coregistered with lateral resolution of approximately $0.5 \mu \mathrm{m}$ and axial resolution of approximately $1.5 \mu \mathrm{m}$. Preliminary studies of a 3-D organotypic epithelial tissue model show that multiphoton images of fluorescence and second harmonic signals are derived from cellular and extracellular matrix structures, respectively, while OCT images are generated from scattering interfaces due to tissue variations in refractive index. The combined MPM/OCT microscope is capable of providing simultaneous functional and structural information from cells and extracellular matrix and is potentially a powerful tool for studying biological processes in thick tissues. () 2006 Society of Photo-Optical Instrumentation Engineers. [DOI: 10.1117/1.2193428]
\end{abstract}

Keywords: multiphoton microscopy; two-photon excited fluorescence; second harmonic generation; optical coherence tomography; femtosecond laser; engineered tissue.

Paper 05383LR received Dec. 21, 2005; revised manuscript received Feb. 14, 2006; accepted for publication Feb. 15, 2006; published online Apr. 17, 2006.

High-resolution imaging techniques derived from complementary signals can be combined to provide 3-D structural and functional information about biological tissues. A critical technical challenge for these "multimodality" imaging meth-

*Tel: 949-824-8367; E-mail: bjtrombe@uci.edu ods is to ensure simultaneous, coregistered information content from each image voxel. For example, optical coherence tomography (OCT) and confocal fluorescence microscopy (CM) have been integrated into a single platform ${ }^{1}$ using both coherence and pinhole gating to achieve image coregistration. Recently, conventional OCT and second-harmonic OCT have been combined together to study reflectance and nonlinear properties of samples using coherence gating strategies. ${ }^{2}$

Multiphoton microscopy $(\mathrm{MPM})^{3}$ and $\mathrm{OCT}^{4}$ are capable of noninvasive, high-resolution imaging in thick, scattering biological tissues. Two major signals detected in MPM are twophoton excited fluorescence (TPEF) and second-harmonic generation (SHG). TPEF can be detected from intrinsic sources (e.g., cofactors, proteins) and exogenous fluorophores, while strong SHG signals can be obtained from noncentrosymmetric molecules such as collagen, a common structural protein. OCT detects backscattered light from refractive index discontinuities that occur between tissues of different structure or composition. Using two separate MPM and OCT systems, our previous research on wound healing and oral cancer progression has demonstrated that MPM and OCT can provide complementary structural and functional information. ${ }^{5,6}$ However, this study was performed on two separate systems with different sampling scales so that coregistration between the MPM and OCT images was difficult.

A combined MPM/OCT system has been previously reported by Beaurepaire et al. ${ }^{7}$ using an 80 -fs Ti:sapphire laser. Because the coherence length of their source was $15 \mu \mathrm{m}$, the intrinsic OCT axial resolution was not matched with the MPM channel. In order to improve the resolution of the OCT channel, a pinhole was inserted into the beam path before the OCT detector, which resulted in significant signal loss. An alternative approach is to employ a source with a much shorter pulse duration and broader bandwidth. Under these conditions, the coherence length of the light source is matched with the MPM axial resolution.

Our combined MPM/OCT platform utilizes a 12-fs Ti:sapphire laser with a spectral bandwidth of $100 \mathrm{~nm}$. The experimental setup of the combined MPM/OCT system is shown in Fig. 1. A femtosecond Ti:sapphire laser (Femtolasers) is pumped by an $\mathrm{Nd}: \mathrm{YVO}_{4}$ laser (Coherent). The center wavelength of the Ti:sapphire is $800 \mathrm{~nm}$. Its output first passes through a pair of fused silica Brewster prisms. The prism pair precompensates the dispersion later accumulated from the objective lens and other optics in the beam delivery path. The beam is then reflected backward by a mirror but angled slightly to be separated from the input beam. Afterward, the laser beam is split by a beam splitter into two arms, the sample arm and the OCT reference arm. In the sample arm, the laser beam is raster scanned by two galvanometer mirrors in an en-face mode. The scanned laser beam is expanded by two lenses to fill the back plane of the objective. A tightly focused laser beam is delivered to the sample by the objective.

From the sample, a variety of signals are generated simultaneously, including the TPEF and SHG signals for MPM imaging and the backscattered fundamental signal for OCT imaging. The TPEF and SHG signals are collected by the same objective lens in a backward direction. They are sepa-

1083-3668/2006/11(2)/020502/3/\$22.00 @ 2006 SPIE 


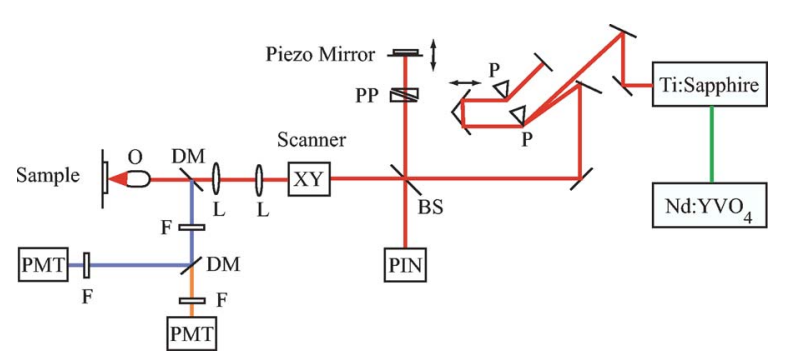

Fig. 1 Schematics of the combined multiphoton and optical coherence microscopy system. BS: beam splitter; DM: dichroic mirror; F: filter; L: lens; O: objective; P: prism; PP: prism pair; PMT: photomultiplier tube.

rated from the excitation source by a dichroic mirror (675DCSP, Chroma). TPEF and SHG are separated by a second dichroic mirror (475DCLP, Chroma) and selected by suitable bandpass filters. The TPEF and SHG signals are detected by two photomultiplier tubes (PMTs), respectively. For OCT imaging, the backscattered fundamental light is reflected by the beam splitter to a PIN detector where it is mixed with the reference arm. In the OCT reference arm, a scanning piezo mirror generates a $5-\mathrm{kHz}$ carrier frequency for OCT detection. In the en-face scanning mode, the scanning range of the piezo mirror is set to be less than the coherence length of the light source so that the OCT signal samples only a thin slice within its axial resolution. A second prism pair in the reference arm provides variable material thickness for balancing the dispersion between the sample and the reference arms. OCT interference fringes are generated on the PIN detector when the path length of the reference arm matches that of the sample arm. The envelope of the interference fringes is demodulated by a lock-in amplifier.

In this combined MPM/OCT system, three channels can simultaneously detect TPEF, SHG, and OCT signals, respectively. The en-face imaging is achieved by raster scanning the two galvanometer mirrors. For $\mathrm{Z}$ scanning, the sample is mounted on a linear translation stage which moves up and down so that there is minimal disruption of optical path length when taking stacks of images in three dimensions. The frame size is $256 \times 256$ pixels. For image acquisition, the pixel dwell time is set at $100 \mu \mathrm{s}$, which is limited by the carrier frequency of the OCT interference fringes.

Due to their nonlinear nature, the MPM signals, including TPEF and SHG, are excited from the focal volume of the laser beam. Therefore, their transverse and axial resolutions are determined by the focal diameter and the focal depth of the objective lens, respectively. For efficient MPM excitation, a water immersion Achroplan $63 \times$ objective (Zeiss) of 0.95 numerical aperture (NA) is used. The theoretically estimated focal diameter is $\sim 0.5 \mu \mathrm{m}$ and the focal depth is $\sim 1.5 \mu \mathrm{m}$. In OCT imaging, the transverse resolution is also given by the focal diameter of the objective. However, axial resolution is derived from coherence gating and defined by the coherence length of the light source. Therefore, it is crucial to select the right light source with a coherence length comparable to the focal depth of the high NA objective. In our case, we used an ultrafast Ti:sapphire laser with a pulse width of $12 \mathrm{fs}$ and a bandwidth of $\sim 100 \mathrm{~nm}$. This bandwidth corresponds to a theoretically estimated round-trip coherence length of

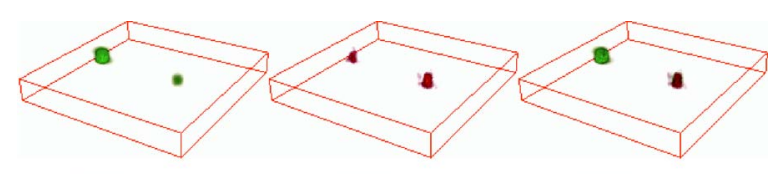

(a)

(b)

(c)

Fig. 2 Three-dimensional reconstruction of MPM/OCT images of 1 - $\mu$ m-diameter fluorescent beads. (a) TPEF image; (b) OCT image; (c) merged image. The image dimension is $20 \times 20 \times 2.5 \mu \mathrm{m}$.

$2.8 \mu \mathrm{m}$, assuming a Gaussian spectral shape. ${ }^{8}$ Thus the estimated axial resolution from coherence gating is $2.8 \mu \mathrm{m}$ in free space and $2 \mu \mathrm{m}$ in agarose gel with a refractive index of $\sim 1.4$.

By using a 12-fs, broadband Ti:sapphire laser source, MPM and OCT resolution can be matched without the need of a pinhole. Ultrashort pulses also provide highly efficient excitation of nonlinear MPM signals because of their high peak power. Dispersion of the ultrashort pulse is precompensated by the Brewster prism pair, resulting in sub-20-fs pulses with the full 100-nm bandwidth at the focal plane. The pulse width is measured at the sample location using a previously reported autocorrelation approach that monitors the SHG intensity generated at the focus while scanning the time delay between two optical pulses. ${ }^{9}$

In order to demonstrate MPM-OCT image co-registration, $1-\mu \mathrm{m}$ fluorescent microspheres (Molecular Probes) suspended in an agarose gel are scanned by the combined MPM/ OCT system. Figures 2(a) and 2(b) show the 3-D reconstruction of the microspheres in the TPEF and OCT channels, respectively. Figure 2(c) shows the merged image from TPEF and OCT. The image dimension is $20 \times 20 \times 2.5 \mu \mathrm{m}$. Locations of the microspheres are well matched in the MPM and OCT channels.

In order to obtain more accurate measurements of MPM and OCT resolution, we acquired three-dimensional image stacks of $0.3-\mu \mathrm{m}$-diameter fluorescent microspheres (Polysciences) suspended in an agarose gel. Figures 3(a) and 3(b) show the transverse and axial point spread functions of the MPM and OCT channels, respectively. In Fig. 3(a) the transverse PSFs from the MPM and OCT channels match closely and their full widths at half maximum (FWHM) are measured to be $\sim 0.5 \mu \mathrm{m}$. In Fig. 3(b) the axial PSFs for MPM and OCT channels are compared. The data are reconstructed from a $\mathrm{Z}$ stack of XY frames. We see a close match between MPM

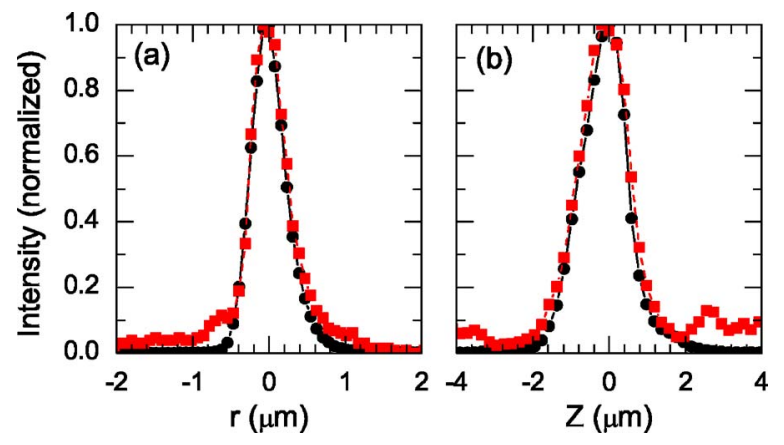

Fig. 3 Transverse (a) and axial (b) point spread functions of MPM and OCT. The circles are for MPM and the squares are for OCT. 


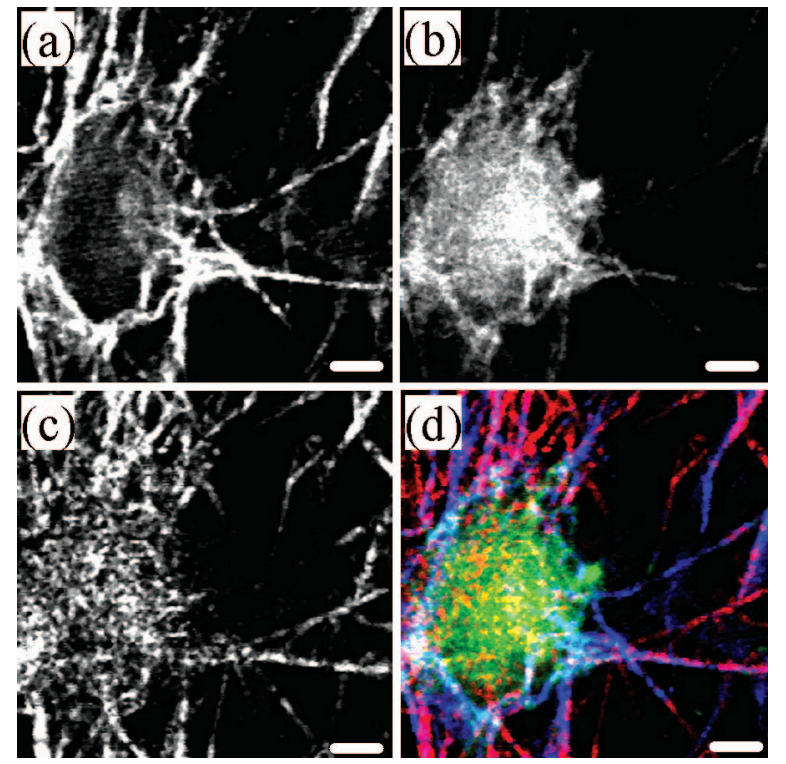

Fig. $4 \mathrm{MPM}$ /OCT images of an organotypic RAFT tissue model. (a) SHG from collagen matrix; (b) TPEF from fibroblasts; (c) OCT from scattering interfaces; (d) merged image with SHG, TPEF, and OCT signals in blue, green, and red. The scale bar is $5 \mu \mathrm{m}$.

and OCT, with a measured axial resolution of $\sim 1.5 \mu \mathrm{m}$, a number that corresponds well with the theoretical estimates of $1.5 \mu \mathrm{m}$ based on the focal depth of the objective and $2 \mu \mathrm{m}$ (in agarose gel) based on coherence gating, respectively.

The advantage of combining MPM and OCT is to acquire complementary structural and functional information about biological tissues. Such capability is tested on an organotypic RAFT tissue model. The RAFT model consists of a basic polymerized collagen gel made up of type-I rat-tail collagen and primary human neonatal dermal foreskin fibroblast cells. Figure 4 shows the images in the (a) SHG, (b) TPEF, and (c) OCT channels, respectively. The SHG channel shows the organization of the collagen matrix with an exclusion of the cell body, the TPEF channel shows the autofluorescence signal from the cell body of a fibroblast, and OCT shows the morphology of the RAFT including the extracellular collagen matrix and the cell. A merged image of the three channels is displayed in Fig. 4(d) with SHG, TPEF, and OCT in blue, green, and red colors, respectively.

The combination of the three channels provides a more complete picture of the tissue with both structural and compositional information that appear to be complementary. For example, more collagen fibers are observed in the OCT vs SHG channels [Fig. 4(c) vs 4(a)], possibly due to the sensitivity of SHG to collagen fibers oriented parallel to the linear polarization of the laser beam. In contrast, all scattering structures will contribute to the OCT signal. Complex patterns observed from the cell body area in the OCT image appear to be scattering, or possibly speckle, from subcellular structures.

The ultrashort pulses provide desirable high peak intensity for MPM and broad bandwidth for high-resolution OCT, respectively. However, the bandwidth also affects the MPM generation. In TPEF, broad bandwidth can reduce the efficiency of two-photon absorption if the laser spectrum is broader than the fluorophore absorption feature. For SHG, broad bandwidth can make it difficult to phase-match all the spectral components. However, in practical terms, the phase matching condition is not critical for SHG interactions over the microscopy length scale (i.e., focal depths $<20 \mu \mathrm{m}$ ). Therefore, the main limitation to the use of increasingly short pulses is probably the efficiency of TPEF generation. Depending on the application, a pulse width can be selected that allows one to balance optimal performance for TPEF, SHG, and OCT, respectively.

In summary, a combined MPM/OCT system is built on a single platform using an ultrashort Ti:sapphire laser that provides efficient MPM excitation and the broad bandwidth required for high-resolution OCT. While their optical sectioning mechanisms are different, we demonstrate that resolution in both transverse and axial directions are closely matched, allowing simultaneous acquisition of coregistered images from MPM and OCT channels. Initial performance is demonstrated on an organotypic RAFT tissue model where extracellular matrix structure, cellular fluorescence, and scattering interfaces are clearly observed in the SHG, TPEF, and OCT channels, respectively. It is expected that the combined MPM/OCT system will provide a valuable tool for studying physiological processes such as cancer and wound healing that involve ECM remodeling, cell migration, and angiogenesis without the use of exogenous probes.

\section{Acknowledgment}

We thank Drs. Enrico Gratton, Chung-Ho Sun, and Alvin Yeh for helpful discussions and assistance. This work was supported by the National Institutes of Health Laser Microbeam and Medical Program (LAMMP-P41RR01192), the Air Force Office of Scientific Research (MFEL program), the Beckman Foundation, and the Whitaker Foundation.

\section{References}

1. J. P. Dunkers, M. T. Cicerone, and N. R. Washburn, "Collinear optical coherence and confocal fluorescence microscopes for tissue engineering," Opt. Express 11(23), 3074-3079 (Nov. 2003).

2. Y. Jiang, I. Tomov, Y. Wang, and Z. Chen, "Second-harmonic optical coherence tomography," Opt. Lett. 29(10), 1090-1092 (May 2004).

3. W. Denk, J. H. Strickler, and W. W. Webb, "Two-photon laser scanning fluorescence microscopy," Science 248, 73-76 (Apr. 1990).

4. D. Huang, E. A. Swanson, C. P. Lin, J. S. Schuman, W. G. Stinson, W. Chang, M. R. Hee, T. Flotte, K. Gregory, C. A. Puliafito, and J. G. Fujimoto, "Optical coherence tomography," Science 254(5035), 1178-1181 (Nov. 1991).

5. A. T. Yeh, B. S. Kao, W. G. Jung, Z. P. Chen, J. S. Nelson, and B. J Tromberg, "Imaging wound healing using optical coherence tomography and multiphoton microscopy in an in vitro skin-equivalent tissue model," J. Biomed. Opt. 9, 248-253 (Mar./Apr. 2004).

6. P. Wilder-Smith, T. Krasieva, W. G. Jung, J. Zhang, Z. P. Chen, K Osann, and B. Tromberg, "Noninvasive imaging of oral premalignancy and malignancy," J. Biomed. Opt. 10, 051601 (Sep./Oct. 2005).

7. E. Beaurepaire, L. Moreaux, F. Amblard, and J. Mertz, "Combined scanning optical coherence and two-photon-excited fluorescence microscopy," Opt. Lett. 24(14), 969-971 (Jul. 1999).

8. A. F. Fercher, W. Drexler, C. K. Hitzenberger, and T. Lasser, "Optical coherence tomography-principles and applications," Rep. Prog. Phys. 66, 239-303 (Feb. 2003).

9. M. Müller, J. Squier, and G. J. Brakenhoff, "Measurement of femtosecond pulses in the focal point of a high-numerical-aperture lens by two-photon absorption," Opt. Lett. 20, 1038-1040 (May 1995). 